DOI: 10.3901/JME.2020.07.052

\title{
浮游式电力变压器内部巡检机器人*
}

\author{
冯迎宾 1,2 于 洋 1,2 高宏伟 1,2 李智刚 ${ }^{3}$ \\ (1. 沈阳理工大学智能与网络化测控技术辽宁省重点实验室 沈阳 110059; \\ 2. 沈阳理工大学自动化与电气工程学院 沈阳 110059; \\ 3. 中国科学院沈阳自动化研究所 沈阳 110016)
}

\begin{abstract}
摘要: 针对变压器间接故障检测方法和人工检测手段存在的问题, 提出了浮游式变压器内部巡检机器人。详细阐述了变压器 内部结构特点, 归纳了机器人应具备的特性。利用水下机器人密封技术设计了机器人球形密封舱体, 机器人采用喷射推进方 式运动, 使机器人具备零回转半径、运动灵活的特点。利用电磁传播理论分析了无线信号在变压器油特殊介质传输的可行性, 并设计了机器人控制系统详细方案, 阐述了机器人搭载的关键传感器及设计了完善的机器人电源管理系统。最后, 搭建了机 器人原理样机, 并在试验油池和 $220 \mathrm{kV}$ 变压器内部开展了示范应用。试验结果表明, 机器人可代替人工在特殊的变压器内 部环境下完成故障巡检任务。
\end{abstract}

关键词: 机器人; 油浸式变压器; 无线通信; 故障检测; 机器人密封

中图分类号: TP242

\section{Floating Robot Design Method for Inspecting the Inside of Transformer}

\author{
FENG Yingbin ${ }^{1,2}$ YU Yang ${ }^{1,2}$ GAO Hongwei ${ }^{1,2}$ LI Zhigang ${ }^{3}$
}

(1. Key Laboratory of Intelligent and Networked Measurement and Control Technology,

Shenyang Ligong University, Shenyang 110059;

2. School of Automation and Electrical, Shenyang Ligong University, Shenyang 1100159;

3. Shenyang Institute of Automation Chinese Academy of Sciences, Shenyang 110016)

\begin{abstract}
A floating robot design method for inspecting the inside of transformer is proposed to address the problem, which exists in the indirect fault detection approach or the artificial detection method. The internal structure characteristics of the transformer are elaborated, and the characteristics of the robot should be summarized. By using the sealing technology of underwater robots, a robot with the spherical structure is designed, where the robot motion is achieved by jet propulsion. The robot has the characteristics of zero radius of gyration and flexible movement. The feasibility of wireless signal transmission in special transformer oil medium is analyzed with electromagnetic wave theory, and the detailed scheme of the robot control system is designed. The key sensors on the robot are described, and a complete power management system is designed. The principle prototype of robot is designed; the robot is demonstrated in the test oil pool and two $220 \mathrm{kV}$ transformer. The experiment results show that the developed method can take the place of the artificial detection method to complete the fault detection tasks under the special internal circumstance of the transformer.
\end{abstract}

Key words: robot; oil-immersed transformer; wireless communications; fault detection; robot seal

\section{0 前言}

变压器在电力系统中承担电压变换、电能分配 和传输的功能, 其运行状态直接影响电力系统的稳

* 中国博士后科学基金面上(2019M661127)和辽宁省自然科学基金 (2019-ZD-0250)资助项目。20190530 收到初稿, 20191029 收到修 改稿
定性与可靠性 ${ }^{[1]}$ 。变压器故障模式因型号、电压等 级、故障部位、故障能量密度、运行状态等不同, 故障形式及表现特征复杂多变, 同时变压器趋向于 大型化、复杂化方向发展，其复杂程度、集成性越 来越高, 各部件之间的耦合程度大大增加, 使其故 障诊断和预测的难度随之增加 ${ }^{[2-3]}$ 。在电力系统运行 维护过程中, 及时准确地判断变压器故障类型、定 位故障点及查找故障发生原因, 是电力部门制定维 
修计划的关键环节。

目前, 变压器故障检测方法主要依据反映变压 器状态的特征信号进行判断, 分为气象色谱在线检 测技术、红外光谱技术、传感器阵列在线检测技术、 变压器油温检测技术等 ${ }^{[4-5]}$ 。由于变压器结构复杂、 故障不确定因素较多, 特征信号反映故障存在一定 的局限性, 学者们陆续提出了基于专家系统、模糊 推理、粗糙集理论、支持向量机、模糊 Petri 网络、 神经网络等人工智能综合故障诊断方法 ${ }^{[6-10]}$ 。以上 这些方法均属于间接故障诊断方法, 不能给出精确 的故障点位置。当前, 变压器内部故障点定位手段主 要依赖于人工, 每次检修需要将变压器油全部排出, 由检修人员进入变压器内部进行检查, 人工检测手段 存在风险高、效率低、易产生二次污染等问题。

本文融合水下机器人密封技术、人机协同控制 技术、密闭充油空间的无线传输技术, 提出了一套 可取代人工检测的浮游式变压器内部巡检机器人设 计方案, 有助于推动变压器内部巡检向自动化、智 能化方向发展。

\section{1 机器人设计需求分析}

\section{1 变压器组成及内部结构特点}

油浸式变压器结构分为器身、油箱、调压装置、 冷却装置、保护装置、出线装置。油箱是指油浸式 变压器的外壳, 油箱内注满变压器油, 变压器油主 要用于绝缘、散热、消弧。变压器内部结构如图 1 所示, 其特点是: 铁心和绕组位于油箱中心, 油箱 内壁与绕组之间的空间狭小; 变压器内部电缆密布, 并且电缆走向不规则; 变压器油箱内部充满变压器 油。因此, 机器人应具备在狭窄空间、结构复杂、 充满变压器油的环境下灵活运动的能力。

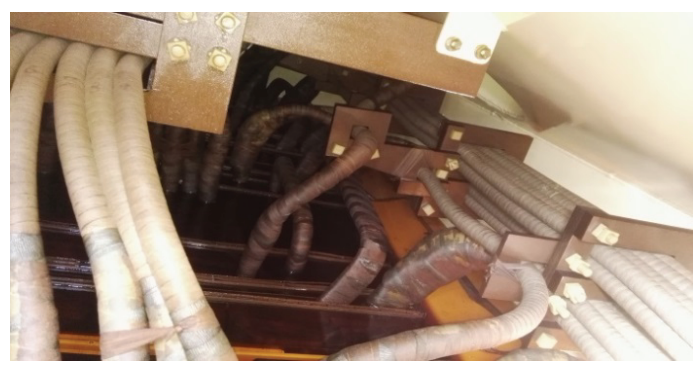

图 1 变压器内部结构

\section{2 机器人需要具备的特性}

变压器油箱的顶部通常存在手孔, 机器人可以 通过手孔, 进入变压器内部。为实现对变压器内部 绕组、底部接线端子、顶端接线端子的全方位观测, 机器人需具备在变压器内部狭窄空间灵活运动的能
力。机器人进入变压器开展观测作业时, 不能造成 变压器油污染, 充分保障变压器的安全性。综合变 压器内部环境特点及观测任务需求, 机器人应具备 的特性在表 1 中列出。

表 1 机器人需具备的特性

\begin{tabular}{cc}
\hline $\begin{array}{c}\text { 机器人设 } \\
\text { 计需求 }\end{array}$ & 解决措施 \\
\hline 防爆性 & 机器人采用锂电池供电, 需具备完善的电池管理系统。 \\
防腐性 & 机器人在变压器油中运动, 外壳需防止变压器油腐蚀。 \\
密封性 & 防止变压器油进入机器人内部。 \\
容错设计 & 机器人具备故障诊断能力, 故障发生时机器人可依靠正 \\
无线传输 & 避免电缆缠绕, 增强机器人灵活运动能力。 \\
多自由度 & 矢量布置推进系统, 水平面具备三个自由度运动能力。 \\
运动 & 采用球形结构设计, 具有零转弯半径、易于脱困的特点。 \\
球形设计 & 通过搭载的高清摄像机及高亮度 LED 实现密闭、无光的 \\
高清视觉 \\
系统
\end{tabular}

\section{2 机器人机械结构设计}

\section{1 机器人结构组成}

为满足机器人在充满变压器油的狭窄空间内部 运动的能力, 机器人采用全封闭的球形结构设计, 具 有零转弯半径、多自由度运动、控制灵活的优点 ${ }^{[11-13]}$ 。 机器人结构如图 2 所示, 分为上罩、中环和下罩部 分, 机器人中环直径 $19 \mathrm{~cm}$ 。在机器人的顶部安装 激光雷达扫描测距模块, 可探测周围障碍物及估计 机器人位置。机器人的上罩布置通信模块、控制板、 垂直喷射洜; 中环布置摄像头、LED 灯、水平喷射 原、深度计; 下罩布置锂电池、电池管理单元、单 轴光纤陀螺、配重铅块等。

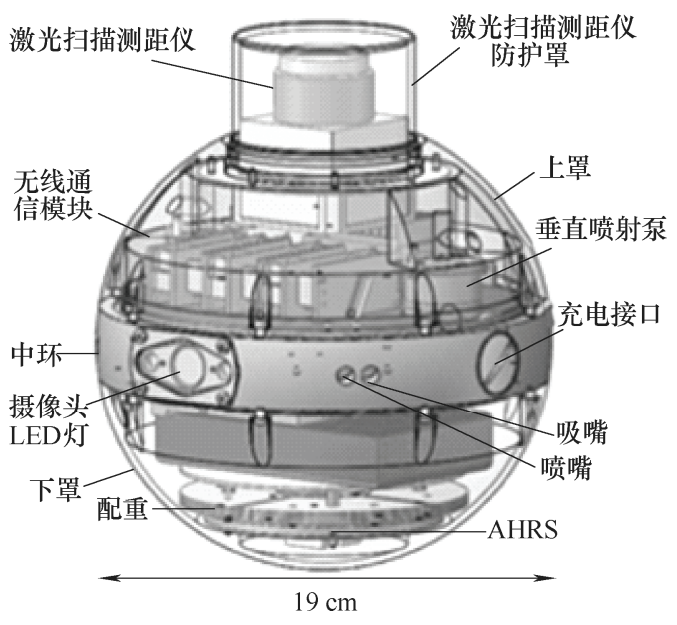

图 2 机器人结构

机器人上罩、下罩、激光扫描测距模块采用聚 碳酸酯材料, 具有耐热、抗冲击、阻燃等性能, 同 
时该材料具有良好的耐变压器油腐蚀的特性 ${ }^{[14]}$ 。机 器人中环采用 6061 铝合金材料, 耐变压器油腐蚀且 有利于内部热量及时导出。上罩、下罩与中环之间 采用 $\mathrm{O}$ 型圈密封结构, 如图 3 所示, 该密封方式结 构简单、可靠性高, 满足机器人密封性、紧凑性的 设计要求。为优化机器人内部空间, 喷射洜采用法 兰安装方式, 泵体直接与喷、吸口相连, 法兰端面 间通过压紧 $O$ 型密封圈实现密封, 如图 4 所示。 $O$ 型密封圈材料选用耐油、耐高温丁腈橡胶, 材料本 身与变压器油不发生反应, 稳定可靠 ${ }^{[15-16]}$ 。

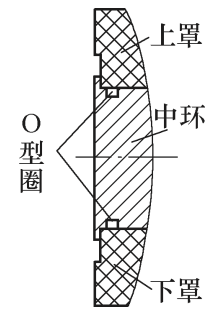

图 3 中环密封结构

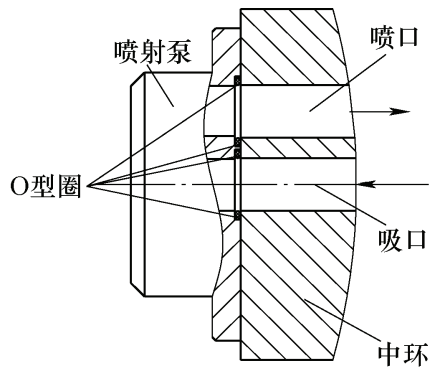

图 4 喷射洜密封结构

\section{2 推进系统设计及受力分析}

由于机器人在充油环境下运动, 推进系统采用 微型喷射洜推进, 该方式不会产生气泡, 可保证变 压器油的清洁性。为保证机器人具有多个自由度灵 活运动的能力, 在机器人的中环矢量布置四个喷射 泵, 结构如图 5 所示, 使机器人具备横移、前后、 旋转三自由度运动能力; 垂直方向布置两个喷射㫤, 结构如图 6 所示, 使机器人具备下潜的能力, 由于 喷射百不能反转, 当机器人上浮时, 需要关闭喷射 泵, 机器人依靠正浮力上浮。

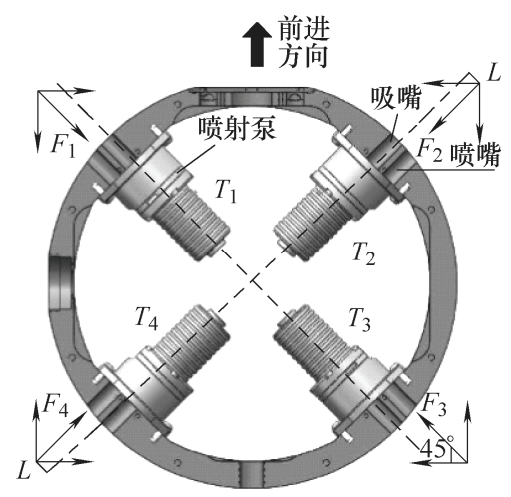

图 5 喷射洜水平安装及受力分析

由于机器人外形结构采用球形设计, 机器人前 后、左右都是轴对称的, 同时机器人水平喷射洜呈 矢量布置, 因此机器人在左移、右移、前进、后退 所受到的阻力和推力大小相同。以机器人前进运动 所受的力为例说明机器人所受喷射洜的推力, 当喷 射百 $T_{3}$ 与 $T_{4}$ 喷射时, 其反作用力 $F_{3}$ 与 $F_{4}$ 的合力使
机器人前进, 其推力大小如式(1)所示

$$
F_{\text {forward }}=\left(F_{3}+F_{4}\right) \cos 45^{\circ}=\frac{\sqrt{2}}{2}\left(F_{3}+F_{4}\right)
$$

由于机器人外形结构采用球形设计, 机器人逆 时针旋转与顺时针旋转所受的力大小相等、方向相 反。喷射葲 $T_{2}$ 与 $T_{4}$ 产生的合力矩使机器人顺时针 旋转, 合力矩大小分别为

$$
M_{\text {clockwise }}=\left(F_{2}+F_{4}\right) L
$$

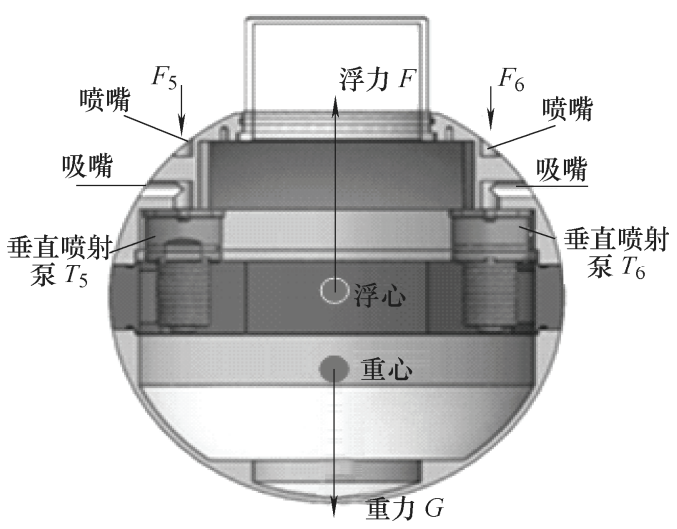

图 6 喷射百垂直安装及受力分析

由于机器人外形结构采用球形设计, 机器人逆 时针旋转与顺时针旋转所受的力大小相等、方向相 反。喷射百 $T_{1}$ 与 $T_{3}$ 产生的合力矩使机器人逆时针 旋转, 合力矩大小为

$$
M_{\text {anti }}=\left(F_{1}+F_{3}\right) L
$$

机器人在垂直方向主要受到推力、浮力、重力 及水动力作用。当机器人全部浸没在变压器油中时, 其正浮力 $F_{z}$ 及垂直喷射洜的推力 $F_{\text {vertical }}$ 可表示为

$$
\begin{gathered}
F_{z}=F_{f}-G \\
F_{\text {vertical }}=F_{5}+F_{6}
\end{gathered}
$$

当喷射百 $T_{5}$ 与 $T_{6}$ 产生的推力之合 $F_{\text {vertical }}$ 大于 $F_{\mathrm{z}}$ 时, 机器人下潜。反之, 则机器人上浮。

\section{3 机器人流体动力学分析}

机器人流体动力学分析是机器人设计的前提 ${ }^{[17-18]}$ 。 本文采用 ANSYS FLUENT 软件对机器人在变压器 油内部的运动特性进行了仿真分析, 得到了机器人 在预定速度下的流体阻力如表 2 所示, 为机器人喷 射洜的选取提供了理论依据。

表 2 阻力表

\begin{tabular}{cc}
\hline 参数 & 数值 \\
\hline 运动速度 $/(\mathrm{m} / \mathrm{s})$ & 0.15 \\
压差阻力 $/ \mathrm{N}$ & 0.165 \\
粘性阻力 $/ \mathrm{N}$ & 0.068 \\
总阻力 $/ \mathrm{N}$ & 0.233 \\
\hline
\end{tabular}




\section{3 机器人控制系统设计}

\section{1 机器人控制系统总体方案}

机器人控制系统采用分布式结构设计, 如图 7 所示, 分为机器人本体控制系统和操作控制终端。 为保障机器人在变压器内部运动的灵活性和安全 性, 控制系统数据传输采用无线通信方式。通信模 块采用 DDL2350 模块, 该模块具有两路 RS232 串 口、1 路 HDMI 高清接口、1 路以太网接口, 可同 时传输高清视频、激光雷达数据、控制指令数据, 具备超低延时、传输距离远、数据加密的优势。机 器人本体控制系统主要包括激光扫描测距模块、无 线通信模块、高清摄像机、核心控制系统、电源管 理单元、航姿参考系统(AHRS)、深度计等。控制系 统的控制单元采用功耗低、接口丰富的 MSP430 单 片机设计, 具有两路 TTL 串口、2 路 AD 采集接口、 2 路 SPI 总线。利用串口芯片 MAX232 将单片机的 $\mathrm{TTL}$ 电平转换为 RS232 电平, 与无线传输模块和

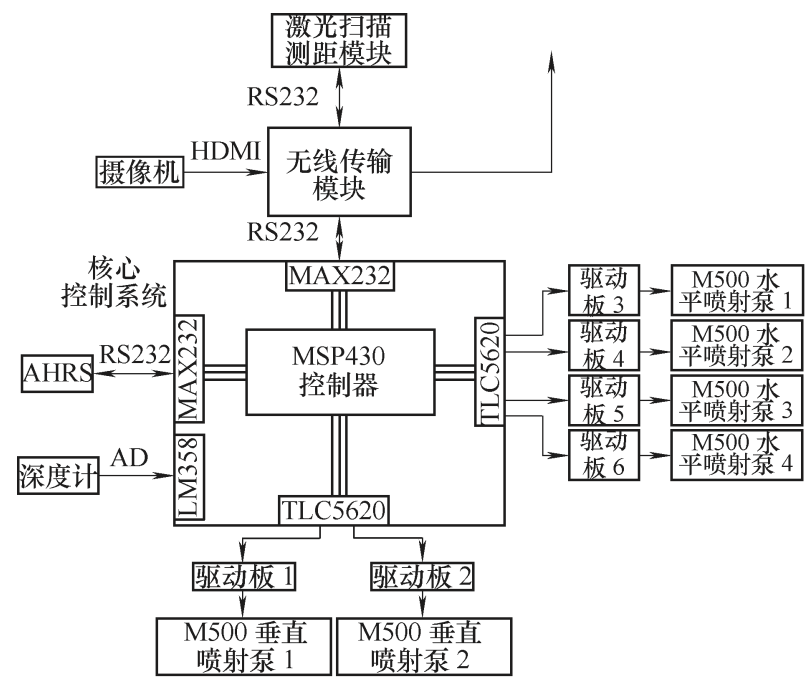

(a) 机器人本体控制系统

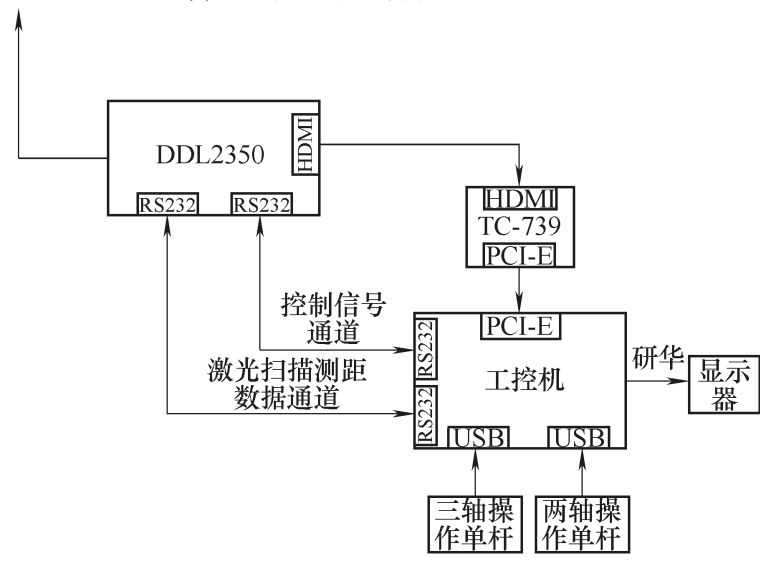

控制端

(b) 机器人操作控制终端

图 7 机器人控制系统总体方案
AHRS 通信。利用 SPI 总线和 TLC5620 芯片扩展出 6 路 DA 接口, 驱动喷射推进泵, 利用 $0 \sim 5 \mathrm{~V}$ 模拟 电压调节喷射洜速度。MSP430 单片机内部集成了 $\mathrm{AD}$ 接口, 深度计输出的模拟量电压经 LM358 芯片 转换后, 进入单片机。

操作控制终端包括微型工控机、显示器、三轴 操作单杆、两轴操作单杆、通信模块、视频采集卡。 操作控制端实时接收、显示、存储激光雷达数据、 高清视频、机器人运行姿态等数据, 同时采集操作 单杆数据, 控制机器人运动速度及方向。

\section{2 机器人传感器系统}

机器人传感器系统由摄像机、深度计、激光扫 描测距模块、AHRS(航姿参考系统)组成, 用于感知 外界环境、测量机器人运行姿态及位置, 如图 8 所 示。摄像机采用 FOXEER 公司的 $4 \mathrm{~K}$ 高清运动相机, 具有体积小、广角大、电子防抖等功能。深度计采 用 GEMS 公司的高精度水下压力传感器, 精度可以 达到厘米级, 提供机器人在变压器中的深度信息。 激光扫描测距模块采用日本北洋公司生产的 URG-04, 可提供机器人在变压器油箱内部的水平位 置信息, 同时可以检测机器人周围的障碍物信息。 航姿参考系统采用 PNI 公司的 AHRS 模块, 集成了 三轴加速度、陀螺仪、三轴磁力计传感器, 经过卡 尔曼滤波数据融合算法输出机器人的运动方向, 具 有抵抗瞬间电磁干扰的能力。

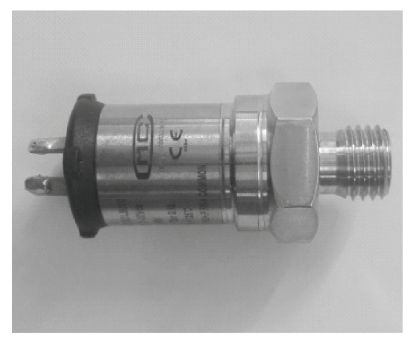

(a) 深度计

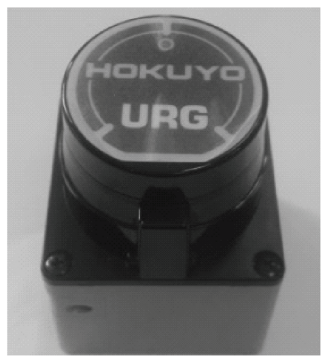

(c) 激光扫描测距模块

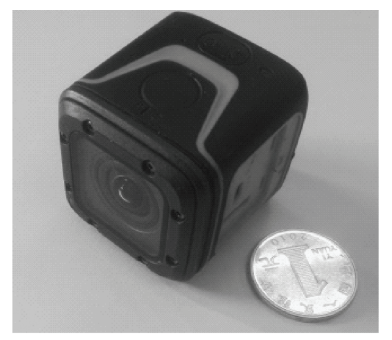

(b) 摄像机

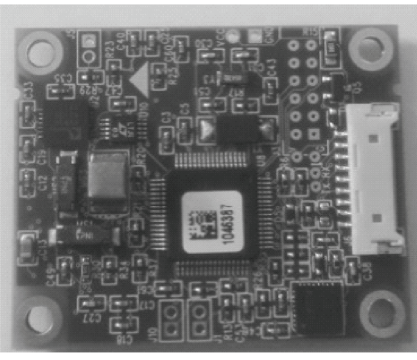

(d) 航姿参考系统
图 8 机器人传感器系统

\section{3 机器人电能管理单元}

机器人采用高能量密度锂电池供电, 为方便机 器人操作和提高机器人安全性, 设计了电能管理系 统和电源保护系统。 
电能管理系统包括磁电开关系统、电源保护系 统。磁电开关系统由继电器和干簧管构成, 是机器 人供电系统的总开关, 原理图如图 9 所示。当永磁 体靠近干簧管 $K_{1}$ 时, $K_{1}$ 闭合, 置位线圈 $S$ 上电, 引 脚 5.6 之间的开关闭合, 锂电池给机器人负载上电; 当永磁体靠近干簧管 $K_{2}$ 时, $K_{2}$ 闭合, 复位线圈 $R$ 上电, 引脚 $5 、 6$ 之间的开关断开, 机器人负载断电。 该系统可利用永磁体在机器人外部控制机器人电源 开关, 无需打开封闭的机器人外壳。

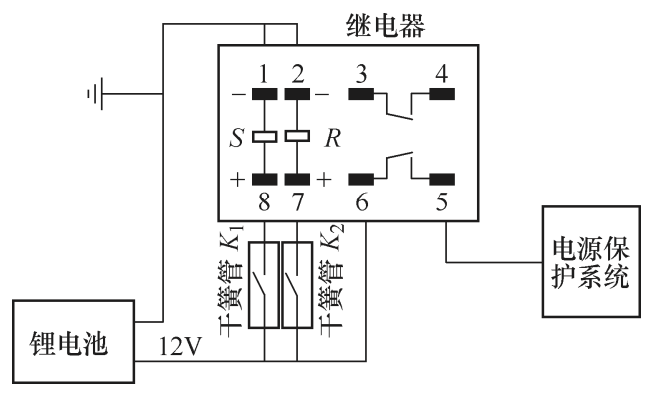

图 9 磁电开关系统

$$
\left\{\begin{array}{l}
R_{1}=\frac{10 \mathrm{~V}-9 \mathrm{~V}}{21 \mu \mathrm{A}}=47.62 \mathrm{k} \Omega \\
R_{2}=\frac{14 \mathrm{~V}-13 \mathrm{~V}}{21 \mu \mathrm{A}}=47.62 \mathrm{k} \Omega \\
R_{3}=\frac{2.5 \mathrm{~V} \times 47.62 \mathrm{k} \Omega}{9 \mathrm{~V}-2.5 \mathrm{~V}}=18.32 \mathrm{k} \Omega \\
R_{4}=\frac{2.5 \mathrm{~V} \times 47.62 \mathrm{k} \Omega}{14 \mathrm{~V}-2.5 \mathrm{~V}}=10.35 \mathrm{k} \Omega \\
R_{S}=\frac{55 \mathrm{mV}}{5 \mathrm{~A}}=11 \mathrm{~m} \Omega
\end{array}\right.
$$

利用 LM5069 芯片设计了电源保护系统, 其原 理图如图 10 所示, 锂电池输出的电流可由电阻器 $R_{\mathrm{s}}$ 测量, 保护系统可根据设置的过压、欠压、电流 保护阈值计算出电阻 $R_{1} 、 R_{2} 、 R_{3} 、 R_{4}$ 。当电流、电 压值超出设定的阈值时, LM5069 芯片关闭输出电 压。系统设置的过压闭锁阈值范围、欠压闭锁阈值 范围、电流闭锁阈值分别为 $13 \sim 14 \mathrm{~V} 、 9 \sim 10 \mathrm{~V}$ 、 5 A。根据 LM5069 芯片手册和设置的电压范围利 用式(6)计算。

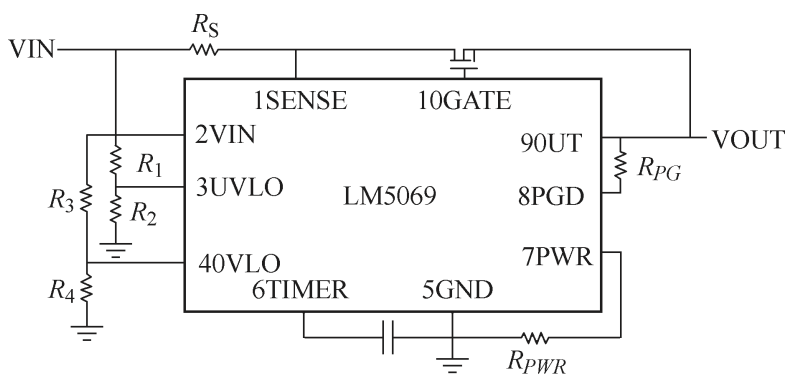

图 10 电源保护系统

\section{4 机器人软件设计}

机器人本体控制系统软件集成了数据接收、传 感器数据采集、数据发送、自主定深、自主定向、 喷射泵控制等功能。此外, 软件利用定时器实时检 测与操作控制端的通信链路, 如通信出现异常, 程 序将关闭所有喷射㬌, 机器人依靠正浮力浮游至变 压器油箱顶部。软件流程图如图 11 所示, 采用中断 的方式接收操作控制端指令, 控制喷射洜运动, 用 轮训的方式依次读取深度传感器数据、AHRS 数据。 数据读取完毕后, 调用数据发送程序, 将机器人运 动姿态发送到操作控制端。当接收到悬停定点观测 指令时, 机器人调用自主定深、自主定向程序。

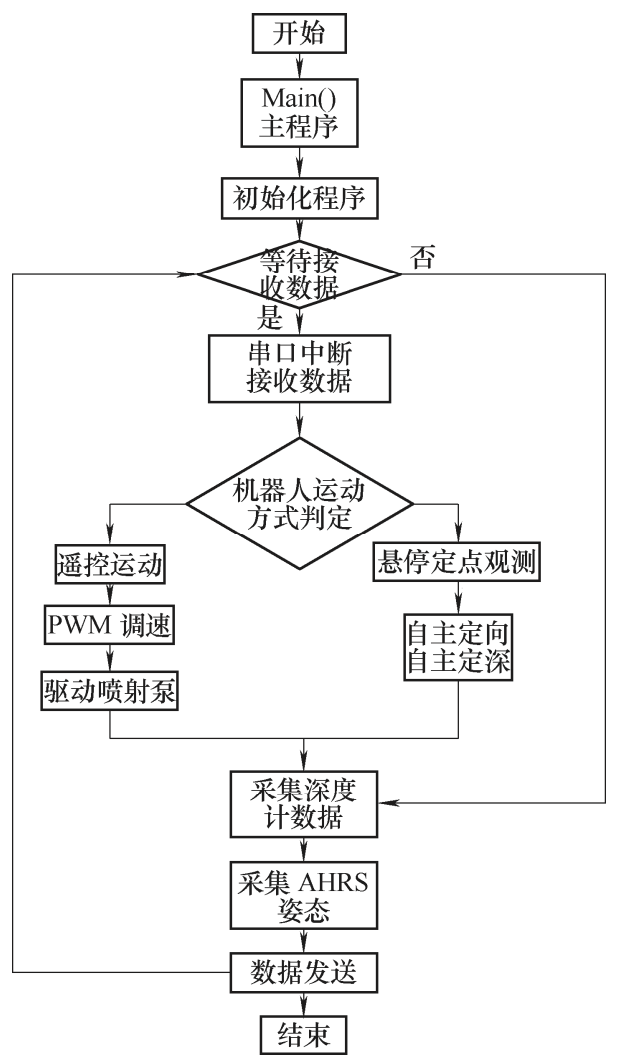

图 11 机器人软件程序流程图

\section{4 无线通信在变压器油传输的} 可行性分析

无线通信系统采用微型高清晰无线传输系统, 该系统采用 $\operatorname{COFDM}($ 编码正交频分复用)多载波调 制技术 ${ }^{[19]}$, 可同时传输 HDMI 视频与 2 路串口数 据, 通信频段为 $2.3 \mathrm{GHz}$ 电磁信号。机器人在变压 器内执行观测任务时, 其电磁信号传输的媒质为变 压器油。

电磁信号在媒质中传播距离取决于在媒质中损 耗的大小 ${ }^{[20]}$ 。媒质的损耗分为介质损耗和焦耳损耗。 
无线通信领域利用 $\sigma / \omega \varepsilon$ 比值将媒质分类, 其 中: $\sigma$ 为电导率、 $\varepsilon$ 为介电常数、 $\omega$ 为角频率, 根 据变压器油参数可知

$$
\frac{\sigma}{\omega \varepsilon}=\frac{1 \times 10^{-12} \frac{\mathrm{S}}{\mathrm{m}} \times \frac{36 \pi}{10^{-9}}}{2 \pi \times 2.3 \times 10^{9} \times 2.5}=3 \times 10^{-12} \ll 10^{-2}
$$

由计算结果可知变压器油属于低损耗介质。电 磁波在低损耗介质的传播损耗可用衰减常数 $\alpha$ 和相 位常数 $\beta$ 表示

$$
\left\{\begin{array}{l}
\alpha=\frac{\omega \sqrt{\mu \varepsilon^{\prime}}}{2}\left(\frac{\varepsilon^{\prime \prime}}{\varepsilon^{\prime}}\right) \\
\beta=\omega \sqrt{\mu \varepsilon^{\prime}}\left[1+\frac{1}{8}\left(\frac{\varepsilon^{\prime \prime}}{\varepsilon^{\prime}}\right)^{2}\right]
\end{array}\right.
$$

式中, $\mu$ 表示磁导率, 介电常数一般用复数表示, $\varepsilon^{\prime} 、 \varepsilon^{\prime \prime}$ 表示介电常数的实部和虚部。对于低损耗介 质, $\varepsilon^{\prime \prime} / \varepsilon^{\prime}=0$ 。

根据变压器油的参数及低损耗介质的特性可以 算出衰减常数及相移常数

$$
\left\{\begin{array}{l}
\alpha=\frac{\omega \sqrt{\mu \varepsilon^{\prime}}}{2}\left(\frac{\varepsilon^{\prime \prime}}{\varepsilon^{\prime}}\right) \approx 0 \\
\beta=\omega \sqrt{\mu \varepsilon}=2 \pi f \frac{\sqrt{\mu_{r} \varepsilon_{r}}}{c}=79.4 \mathrm{rad} / \mathrm{m}
\end{array}\right.
$$

由计算结果可知, 在给定的 $2.3 \mathrm{GHz}$ 频率下, 变压器油媒质具有理想介质的特性, 可以传输无线 信号, 因此机器人在变压器内部可以采用无线通信 方案进行控制。

\section{5 机器人样机试验}

机器人样机、测试油箱和控制终端如图 12 所 示, 首先在变压器油箱中进行了功能性验证。变压 器油箱长宽高分别为 $120 \mathrm{~cm} 、 90 \mathrm{~cm} 、 60 \mathrm{~cm}$, 变 压器油深度 $40 \mathrm{~cm}$ 。机器人操作控制终端包括三轴 单杆、双轴单杆、显示器等, 三轴单杆可控制机器 人前后、横移、旋转运动, 双轴单杆可控制机器人 上升、下潜。

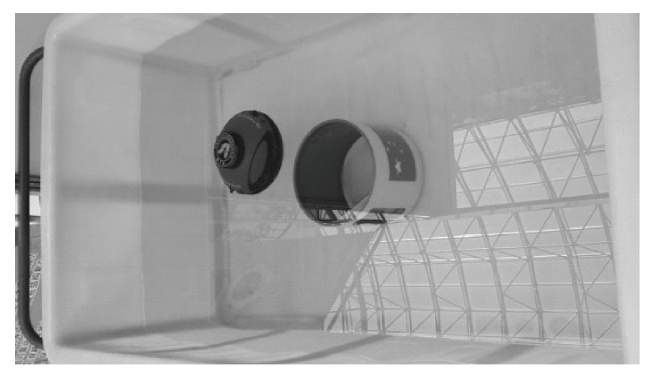

(a) 测试油箱

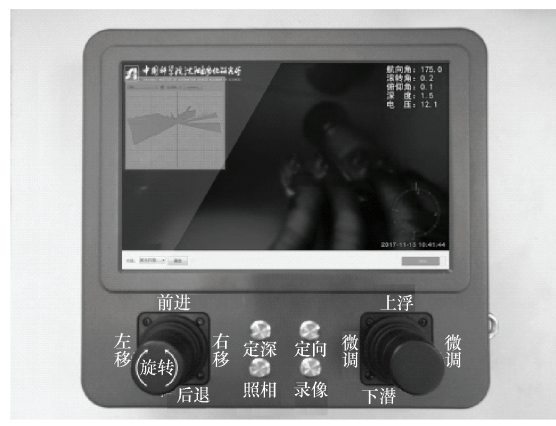

(b) 机器人控制终端

图 12 机器人运动特性测试平台

当逆时针旋转三轴单杆时, 机器人喷射百 $T_{1}$ 、 $T_{3}$ 工作, 旋转速度由单杆的旋转幅值决定, 旋转运 动过程如图 13 所示。机器人旋转过程中深度计的数 据如图 14 所示, 从中可以看出机器人旋转运动对垂 直运动方向的影响可忽略。
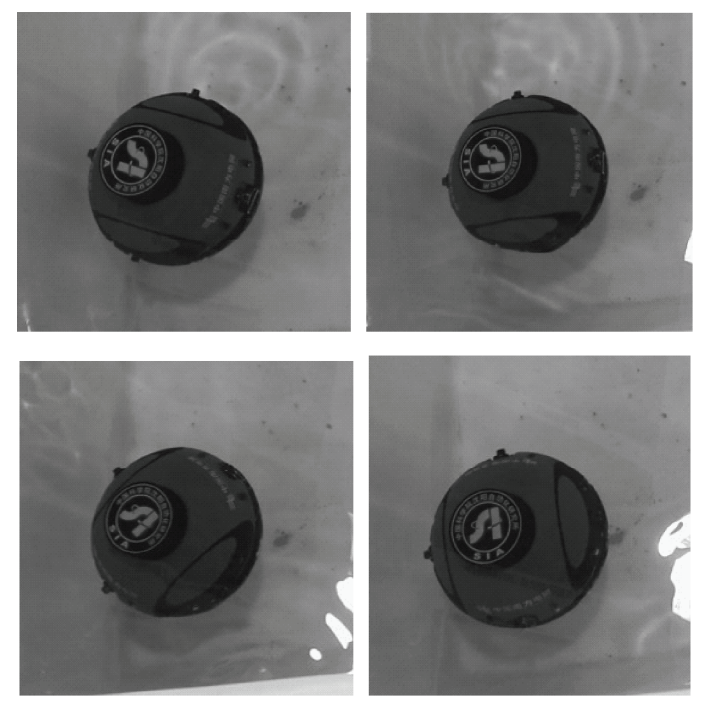

图 13 机器人旋转运动过程

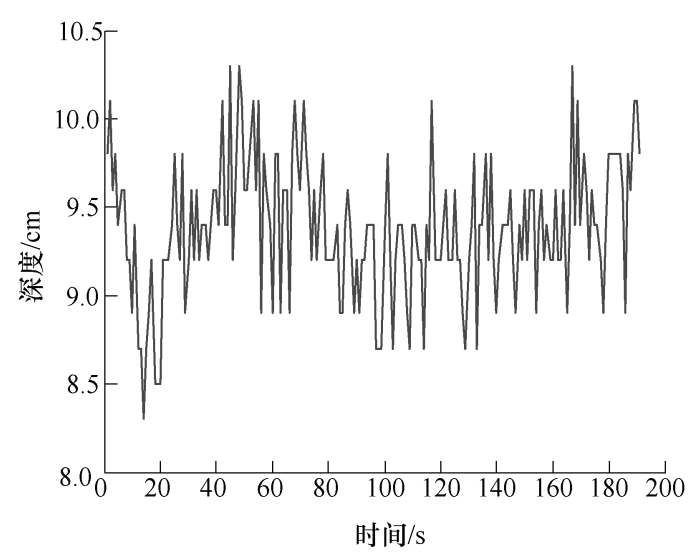

图 14 深度计数据

当向上推动双轴单杆时, 机器人垂直喷射㬌 $T_{5}$ 、 $T_{6}$ 电动机旋转, 旋转速度由单杆的旋转幅值决定, 喷射泵产生的推力大于机器人正浮力时, 机器人下 
潜。由于喷射泵电动机不能反转, 机器人只能依靠 正浮力上浮(图 15)。
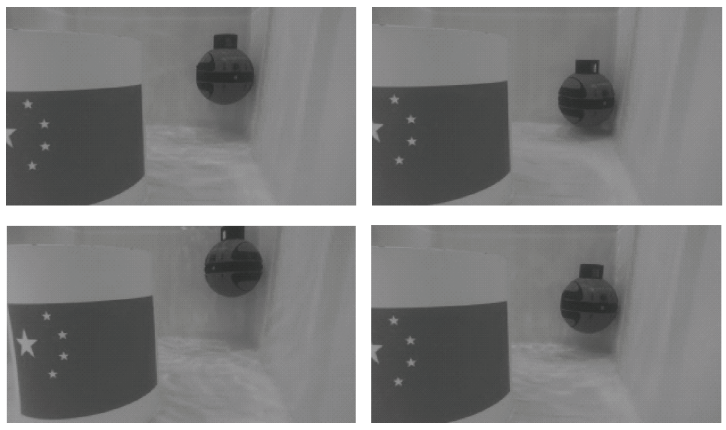

图 15 机器人升沉运动过程

在机器人运动试验基础上, 利用滑膜控制器设 计了机器人定向、定深控制算法, 在变压器油池内 部做了试验, 试验结果如图 16、17 所示。

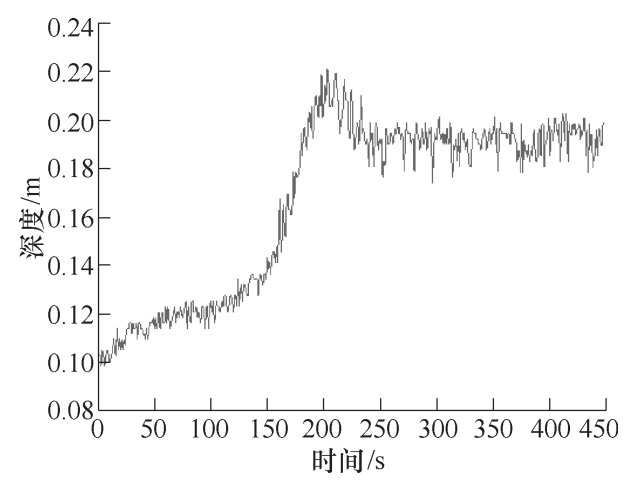

图 16 机器人定深试验调节图

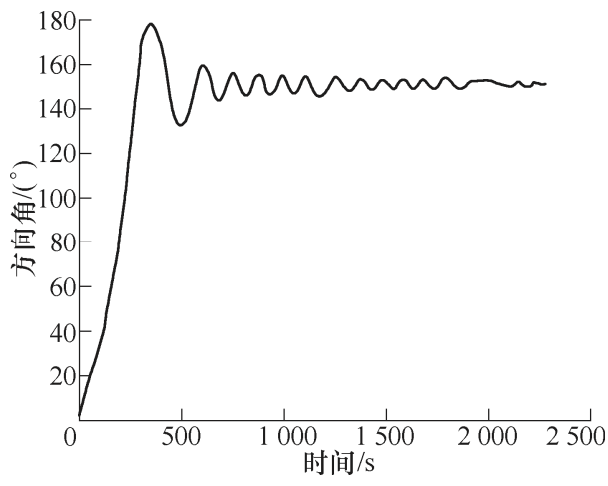

图 17 机器人定向试验调节图

在油池试验的基础上, 机器人进入某变电站 $220 \mathrm{kV}$ 三菱变压器内部, 如图 18 所示, 开展了示范 应用。机器人通过变压器顶部的手孔进入变压器内部。 机器人操控终端显示接收到视频图像数据如图 19 所 示, 通过视频图像可以判断变压器内部是否存在故障。

机器人在变压器内部进行故障巡检与人工巡检 方式相比, 由于不需要维修工人进入变压器内部, 因此不需要进行排油、滤油、注油等繁琐的过程。 以一台 $220 \mathrm{kV}$ 变压器为例, 机器人故障检测方式 仅需要 2 3 名工人, 一天时间即可完成; 而人工巡
检方式需要 8 10 名工人, 三天时间才可完成。因 此, 机器人故障巡检方式在巡检效率和人工成本上 具有明显优势。

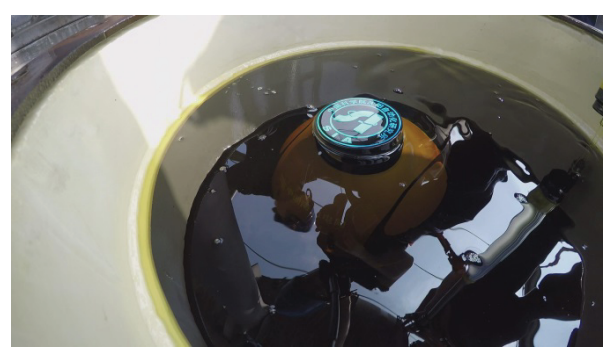

图 18 机器人进入变压器内部

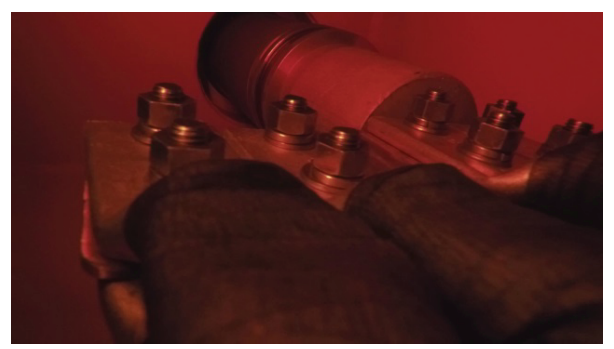

图 19 视频图像

\section{6 结论}

本文介绍了一种浮游式电力变压器内部巡检机 器人。详细分析了变压器内部特殊环境, 总结了机器 人需具备的特性。设计了一种球形机器人外形结构, 机器人具有零回转半径, 多自由度灵活运动的特点。 理论分析了无线通信技术在变压器油特殊介质传播 的可行性, 并利用无线通信技术搭建了机器人控制系 统, 设计了完善的机器人电源管理系统, 保证机器人 在变压器内部安全、可靠运行。最后, 设计了机器人 原理样机, 并在试验油池和变压器内部开展了功能性 试验和示范应用, 试验结果表明: 机器人具有良好的 密封性、耐腐蚀性、运动灵活的特点, 并可获得清晰 的变压器内部图像, 可代替人工完成巡检任务。

\section{参 考 文 献}

[1] SICA F C, GUIMARAES F G, DURATE R O, et al. A cognitive system for fault prognosis in power transformers[J]. Electric Power Systems Research, 2015(127): 109-117.

[2] 邓芳明, 温开云, 何怡刚, 等. 基于 RFID 传感标签及 QPSO-RVM 的变压器绕组故障在线诊断技术 $[\mathrm{J}]$. 中国 电机工程学报, 2018, 38(24): 7183-7193.

DENG Fangming, WEN Kaiyun, HE Yigang, et al. On-line fault diagnosis for transformer windings based on RFID 
sensor tags and QPSO-RVM[J]. Proceedings of The Chinese Society for Electrical Engineering, 2018, 38(24): 7183-7193.

[3] FAIZ J, SOLEIMANI M. Dissolved gas analysis evaluation in electric power transformers using conventional methods a review[J]. IEEE Transactions on Dielectrics \& Electrical Insulation，2017，24(2): 1239-1248.

[4] AYACHIT A, KAZIMIERCZUK M K. Transfer functions of a transformer at different values of coupling coefficient[J]. IET Circuits Devices \& Systems, 2016, 10(4): 337-348.

[5] AIZPURUA J I, CATTERSON V M, STEWART B G, et al. Power transformer dissolved gas analysis through Bayesian networks and hypothesis testing[J]. IEEE Transactions on Dielectrics and Electrical Insulation, 2018, 25(2): 494-506.

[6] 李刚, 于长海, 范辉, 等. 基于多级决策融合模型的电 力变压器故障深度诊断方法 $[\mathrm{J}]$. 电力自动化设备, 2017, 37(11): 138-144.

LI Gang, YU Changhai, FAN Hui, et al. Deep fault diagnosis of power transformer based on multilevel decision fusion model[J]. Electric Power Automation Equipment, 2017, 37(11): 138-144.

[7] 李春茂, 周妹末, 刘亚婕, 等. 基于邻域粗糙集与多核 支持向量机的变压器多级故障诊断[J]. 高电压技术, 2018(11): 3474-3482.

LI Chunmao, ZHOU Momo, LIU Yajie, et al. Multi-level fault diagnosis of transformer based on neighborhood rough set and multiple kernel support vector machine[J]. High Voltage Engineering, 2018(11): 3474-3482.

[8] YU Shenghao, ZHAO Dongming, CHEN Wei, et al. Oil-immersed power transformer internal fault diagnosis research based on probabilistic neural network[J]. Procedia Computer Science, 2016(1): 1327-1331.

[9] HU Sijia, LI Yang, XIE Bin, et al. A Y-D multifunction balance transformer-based power quality control system for single-phase power supply system[J]. IEEE Transactions on Industry Applications, 2016, 52(2): 1270-1279.

[10] HANG Bangcheng. Integrated power and single axis attitude control system with two flywheels[J]. Chinese Journal of Mechanical Engineering, 2012, 25(3): 564-575.

[11] LI Yaxin, GUO Shuxiang, WANG Yu. Design and characteristics evaluation of a novel spherical underwater robot[J]. Robotics and Autonomous Systems, 2017(1): 61-74.

[12] FAN J Z, ZHANG W, KONG P C, et al. Design and dynamic model of a frog-inspired swimming robot powered by pneumatic muscles[J]. Chinese Journal of
Mechanical Engineering, 2017, 30(5): 1-10.

[13] CHANG Jian, LI Bin, WANG Chong, et al. Evaluation method on steering for the shape-shifting robot in different configurations $[\mathrm{J}]$. Chinese Journal of Mechanical Engineering, 2016, 29(1): 21-32.

[14] 张笑笑. 聚碳酸酯复合材料电学和力学性能的研究 [D]. 北京: 北京化工大学, 2013.

ZHANG Xiaoxiao. Study on electrical and mechanical properties of polycarbonate composites[D]. Beijing: Beijing University of Chemical Technology, 2013.

[15] JABER A M Y, MEHANNA N A, OWEIMREEN G A, et al. The effect of DBDS, DBPC, BTA and DBP combinations on the corrosion of copper immersed in mineral transformer oil[J]. IEEE Transactions on Dielectrics \& Electrical Insulation, 2016，23(4): 1-7.

[16] KRZEMINSKA S, RZYMSKI W M, MALESA M, et al. Gloves against mineral oils and mechanical hazards composites of carboxylated acrylonitrile butadiene rubber latex[J]. International Journal of Occupational Safety and Ergonomics, 2016, 22(3): 350-359.

[17] 吴乃龙, 吴超, 葛軕, 等. 基于鱼类体线感知机理的水 下机器人水流场识别研究 $[J]$. 机械工程学报, 2016, 52(13): 54-59.

WU Nailong, WU Chao, GE Tong, et al. Flow recognition of underwater vehicle based on the perception mechanism of lateral line[J]. Journal of Mechanical Engineering, 2016, 52(13): 54-59.

[18] 刘贵杰, 宫华耀, 吴乃龙, 等. 基于鱼类测线感知机理 的流场辨识方法及仿真研究 [J]. 机械工程学报, 2016, 52(17): 162-167.

LIU GUIjie, GONG Huayao, WU Nailong. Simulation research in water condition recognition method based on fish lateral line sensing mechanism[J]. Journal of Mechanical Engineering, 2016，52(17): 162-167.

[19] LIU X, WU H C. Novel Asterisk 16QAM constellation for COFDM[J]. IEEE Communications Letters, 2010, 14(7): 596-598

[20] 张瑜. 电磁波空间传播 $[\mathrm{M}]$. 西安：西安电子科技大学 出版社, 2007.

ZHANG Yu. Electromagnetic wave space propagation[M] Xi'an: Xi'an Dianzi University Publisher, 2007.

作者简介: 冯迎宾, 男, 1986 年出生, 博士。主要研究方向为水下机器 人控制。

E-mail: 781787793@qq.com

于洋(通信作者), 男, 1963 年出生, 硕士, 教授, 硕士研究生导师。主 要研究方向为智能检测技术。

E-mail:824721005@qq.com 